\title{
Aplicación del Modelo de Cobertura de Conjuntos a la Localización de Centros de Distribución de Mercancías dentro de la Ciudad de Pachuca, Hidalgo
}

Montufar-Benítez, Marco A. ${ }^{\mathrm{a}, 1^{*}}$, Montaño-Arango, Oscar ${ }^{\mathrm{a}}$, Corona-Armenta, José R. ${ }^{\text {a }}$, Rivera-Gómez, Héctor ${ }^{\text {a }}$

a Área Académica de Ingeniería y Arquitectura, Instituto de Ciencias Básicas e Ingeniería, Universidad Autónoma del Estado de Hidalgo.

\begin{abstract}
Resumen
El presente trabajo muestra una aplicación del modelo de cobertura de conjuntos, a un problema de localización de centros de abastecimiento de víveres. El crecimiento poblacional de las ciudades en los últimos años, ha provocado un incremento en la demanda de bienes de consumo. En la ciudad de Pachuca, capital del Estado de Hidalgo (México), podemos encontrar una gran cantidad de proveedores de vivieres como: tiendas de conveniencia, mercados, tianguis, además del principal proveedor de mercancías para la población general; la "Central de Abastos" (CA), la cual ha venido causando una problemática debido a su cercanía relativa con colonias altamente pobladas de estatus socioeconómico alto y otras instalaciones de servicio público, como la Central de Autobuses.

En este artículo se presenta una propuesta para la relocalización a nivel macro de la CA, sustituyéndola por centros de distribución (CD’s), ubicados mediante un modelo de optimización, en lugares que satisfacen el criterio de que los habitantes de las distintas colonias de la ciudad no recorren más de una distancia máxima predeterminada para comprar sus víveres. Como primera etapa para la localización de los CD’s, se tomaron treinta colonias de la ciudad, como muestra del estudio y mediante una encuesta en la población; se determinó la preferencia respecto a la distancia máxima que les gustaría recorrer para abastecerse de mercancías. La solución del modelo determinó que deben ser cuatro, el menor número de CD’s localizados en las colonias de: Santiago Tlapacoya, San Pedro Nopancalco, Lomas de Vista Hermosa y Villas de Pachuca.
\end{abstract}

Palabras Clave: Localización optima, Cobertura de conjuntos, Centros de distribución, Programación entera, Ciudad de Pachuca.

\section{Introducción}

La localización de centros de distribución, constituye uno de los factores más importantes para la planeación estratégica de una cadena de suministro. (Chopra y Meindl 2015).

Un centro de distribución es el eje principal de comercialización en una empresa, es el lugar donde las órdenes de pedido se realizan, procesan y despachan al cliente, funciona como punto estratégico para el transporte y almacenamiento de mercancía desde los proveedores hasta los clientes. Una de sus funciones principales, es responder a los cambios que puede haber entre la oferta y la demanda, para el óptimo cumplimiento de un servicio, evitando al máximo el movimiento de productos y proteger los inventarios de robos, pérdidas y daños. (Ravindran y Warsing, 2014).

Cuando se aborda el problema de localización de instalaciones, podemos encontrarnos con preguntas como: ¿Dónde debemos ubicarlo? ¿Cuál es el tamaño óptimo que

\footnotetext{
* Autor en correspondencia.

Correo electrónico: montufar@uaeh.edu.mx (Montufer-Benítez, Marco A.)
}

debe tener? entre otras más. Debido a esto, el problema de localización implica un estudio detallado de necesidades.

Hay dos razones principales por las que las decisiones de localización son una parte muy importante en el diseño del sistema de producción. Una es que implican un compromiso a largo plazo, ya que al cometer un error podría ser difícil de superar, la otra es que las decisiones de ubicación a menudo tienen un impacto en la inversión. Por ejemplo, una mala elección de la ubicación puede dar lugar a un costo excesivo de transporte, pérdida de ventaja competitiva, insuficiencia en suministros de materias primas, o alguna condición similar que sea perjudicial para las operaciones. En el caso de los servicios, una ubicación deficiente podría provocar la pérdida de clientes y/o altos costos de operación.

Los problemas de localización de instalaciones, como ya se mencionó pueden implicar satisfacer varios objetivos, sin embargo, un criterio frecuente consiste en ubicar un conjunto de instalaciones y asignar a estos un conjunto de puntos de demanda o usuarios, de tal manera que sea minimizado el 
costo, para satisfacer la demanda de los usuarios con respecto a algún conjunto de restricciones.

En este trabajo se analizó la manera de optimizar el número de CD's que podrían sustituir a la CA de la ciudad de Pachuca, localizándolos en lugares de la zona conurbada, de tal manera que los usuarios recorran distancias aceptables.

Existen casos documentados acerca de la localización de instalaciones, por ejemplo: (Clemen y Reilly 2001) lo hacen para una biblioteca, (Kirwood 1982) para una planta de energía, (Merkhofer and Keeney 1987) para un sitio de disposición de residuos peligrosos, y (de Neufville and Keeney 1972) para el aeropuerto de la Ciudad de México, donde aplicaron la teoría de la utilidad multi-atributos (MAUT).

\section{Materiales y métodos}

\subsection{Análisis de la problemática}

Pachuca de Soto, es la ciudad capital del Estado de Hidalgo, y de acuerdo con el censo INEGI 2015, la ciudad tenía una población. de 277375 habitantes.

(http://cuentame.inegi.org.mx/monografias/informacion/hgo /poblacion/)

En la ciudad de Pachuca, se puede observar un crecimiento demográfico y económico a lo largo de los años, actualmente la ciudad de Pachuca cuenta con instalaciones que brindan servicios de venta de mercancías para toda la población, comenzando con las tiendas que podemos encontrar en cada esquina de cualquier colonia, pasando por mercados, tianguis y el proveedor popular de insumos que es la Central de Abastos de la ciudad.

El costo de las mercancías en las tiendas y mercados de la ciudad es mayor en comparación del costo que ofrece la CA, sin embargo, algunos consumidores prefieren abastecerse en lugares más cercanos a sus domicilios, y en donde se encuentren otros servicios cercanos, como lo son los centros comerciales. Una primera desventaja de la ubicación actual de la CA, es que se encuentra relativamente cerca de unidades habitacionales de estatus socioeconómico alto, lo cual es percibido como una mala imagen para la zona. Otra desventaja, son los conflictos de flujo vehicular y de personas, razón por la cual los colonos de la zona han generado iniciativas de relocalizar dicha CA (unoTV, julio de 2017).

Observando dicha problemática, en este trabajo se propone la manera de sustituir la actual CA y en su lugar crear CD's, los cuales harían que las personas recorrieran menos distancia, y por lo tanto tendrían un ahorro monetario de transporte. Nuestra búsqueda de información al respecto de intentos por resolver este problema de manera metódica, no condujo a resultados.

\subsection{Aplicación de encuestas}

Para entender como la población asocia el costo de transporte y la distancia que está dispuesto a viajar para abastecerse de víveres, se realizaron encuestas en distintas colonias de la ciudad de Pachuca, para conocer la distancia máxima que las personas están dispuestas a recorrer. Estas encuestas tuvieron valores en kilómetros, teniendo como opciones para escoger de 5,10 o $15 \mathrm{~km}$.

Del análisis de dichas encuestas resultó que las personas eligieron en un $83 \%$ la distancia de $5 \mathrm{~km}$, mientras que un $12 \%$ eligió la opción de $10 \mathrm{~km}$ y un $5 \%$ la opción de $15 \mathrm{~km}$.

Dadas las limitantes de tiempo y presupuesto en este estudio, usamos un muestro de conveniencia o intencional.

Por lo tanto, usamos como cobertura la distancia de $5 \mathrm{~km}$, en el modelo de optimización que se describe en la siguiente sección.

\subsection{Modelo Matemático}

Existen en la literatura modelos matemáticos para resolver problemas de localización; los cuales suelen ser frecuentemente de programación matemática y simulación (Taha, 2017 y Winston, 2018). Dentro de los modelos de optimización, el de cobertura de conjuntos (set covering) se usó, por ejemplo; para ubicar instalaciones de recuperación de desastres (Delke et al., 2005)

Un modelo de cobertura de conjunto, consiste en encontrar el menor número de instalaciones, (en nuestro caso, los CD’s) que por lo menos cubran o abastezcan las necesidades de servicio (colonias de la ciudad, en nuestro caso).

\subsection{1 variables de decisión, restricciones y función objetivo}

Las variables de decisión en este caso, están definidas por el vector columna siguiente:

$$
X_{i}=\left\{\begin{array}{l}
1, \quad \text { si se instala un } C D \\
\text { en la colonia } i, i=1, \ldots, n \\
0, \quad \text { en cualquier caso }
\end{array}\right.
$$

De acuerdo con los resultados de la encuesta, en la cual un alto porcentaje de las personas eligió que la mayor distancia que estaría dispuesta a recorrer para obtener insumos serian 5 $\mathrm{km}$, se seleccionaron 30 colonias con la mayor población y se formó una matriz de 30 x 30, la cual se muestra parcialmente en la figura 1. 


\begin{tabular}{l|l|l|l|l|l|l|l|l|} 
& 1 & 2 & 3 & 4 & 5 & 6 & 7 & 8 \\
\hline 1 & 1 & 0 & 1 & 1 & 0 & 0 & 0 & 0 \\
\hline 2 & 0 & 1 & 0 & 0 & 0 & 0 & 0 & 0 \\
\hline 3 & 1 & 0 & 1 & 1 & 1 & 0 & 0 & 0 \\
\hline 4 & 1 & 0 & 1 & 1 & 1 & 0 & 0 & 1 \\
\hline- & - & - &. &. &. &. &. &.
\end{tabular}

Figura 1: Matriz de alcanzabilidad

La matriz de alcanzabilidad $M_{i, j}$ anterior está formada de forma binaria por unos y ceros en cada posición $(i, j)$, el 1 significa que la colonia i (renglón) se encuentra a una distancia menor o igual a $5 \mathrm{~km}$ de la colonia j (columna), de lo contario hay un 0 .

El proceso de llenado de la matriz anterior se hizo a través del cálculo de distancias a través de la herramienta Google Earth (https://earth.google.com/web), por ejemplo, la Fig. 2 muestra este proceso con dos colonias.

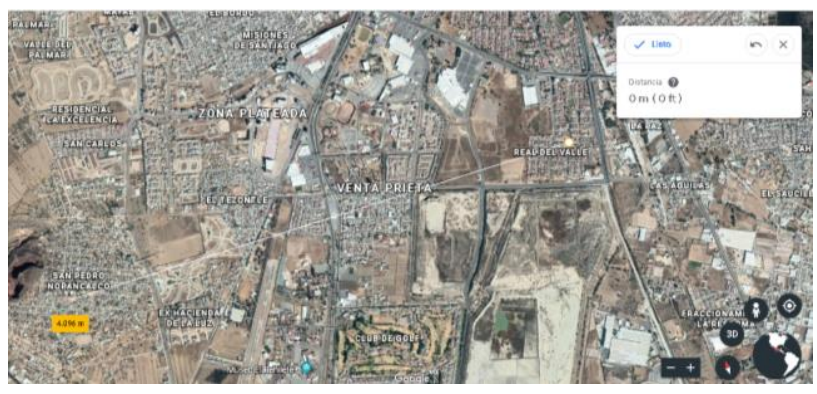

Figura 2: Ejemplo del cálculo de distancias entre colonias

Entonces, con la matriz de alcanzabilidad y el vector de variables de decisión, se construyeron las restricciones de que cada colonia tenía que ser cubierta por lo menos por un CD, ubicado a menos de cinco kilómetros. Estas restricciones puestas en forma matemática se expresan como:

$$
\sum_{j=1}^{j=n} M_{i, j} X_{j} \geq 1, \quad \forall i=1, \ldots, n
$$

Por lo tanto, la función objetivo queda como:

$$
\text { Min: } \quad Z=\sum_{i=1}^{i=n} C_{i} X_{i}
$$

Donde, $C_{i}$ es el costo de instalar un CD en la colonia $i$, en nuestro caso, como se quiere solo minimizar el número de CD's, se seleccionaron estos coeficientes iguales a uno todos. El modelo fue implementado en el complemento Solver de Excel, y arrojó que las colonias en donde se deberían colocar los CD son las siguientes:

\footnotetext{
- Santiago Tlapacoya

- San Pedro Nopalcalco

- $\quad$ Lomas de Vista hermosa
}

- Villas de Pachuca

De esta manera, se puede abastecer a las 30 colonias, sin tener que recorrer más de cinco kilómetros, ayudando así de alguna manera a disminuir el tráfico de personas y automóviles en la zona actual de la CA.

La figura 3, muestra la disposición espacial de estas colonias, con el radio de cinco kilómetros. Como podemos observar la gran mayoría de la población queda cubierta por lo menos por un $\mathrm{CD}$.

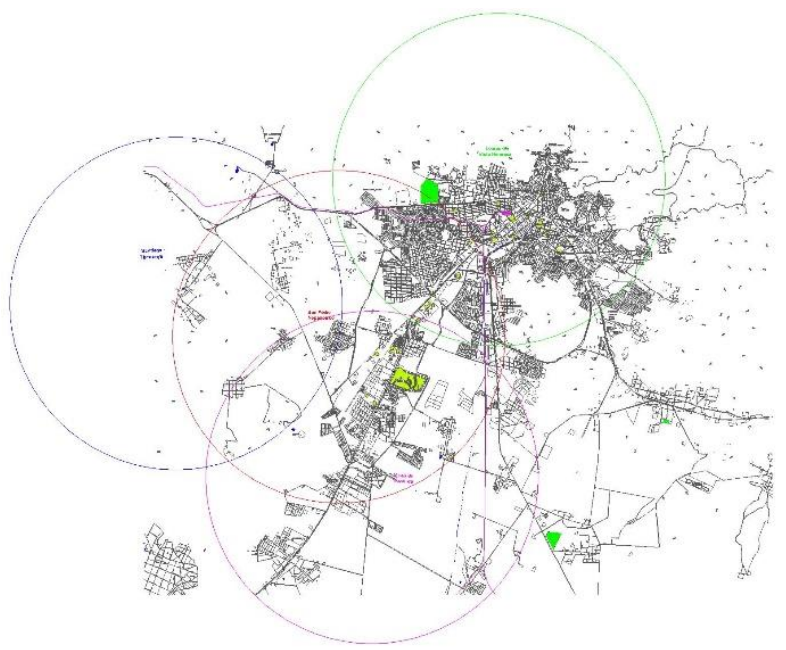

Figura 3. Disposición final de los CD’s

\section{Conclusión y estudios futuros}

Por medio de un modelo de programación entera (cobertura de conjuntos), se pudo determinar que el número óptimo (mínimo) de centros de distribución de mercancías, que podrían sustituir la actual CA, es de cuatro para la ciudad de Pachuca.

Estudios futuros podrían considerar: el costo de las instalaciones nuevas, el flujo de personas (número de viajes) hacia los nuevos centros de distribución, e impacto ambiental en los sitios contemplados. Así también es posible conducir un estudio de sensibilidad proponiendo otras distancias máximas de cobertura.

Los autores agradecen al programa CYTED, el apoyo a la red temática BigDSS-Agro (P515RT0123).

\section{English Summary}

\section{Optimal Location of Consumer Goods Distribution Centers Within the Pachuca City}

\section{Abstract}

The enormous population growth of the cities in recent years has caused an increase in the demand for goods. In the Pachuca city, capital of the State of Hidalgo (Mexico), we can find a large number of stores, markets and tianguis, in addition to the main supplier of supplies for the general 
population; the "Central de Abastos" (CA), which has been causing a problem due to its relative proximity to highly populated colonies of high socioeconomic status and other facilities such as the Bus Terminal.

This article presents a proposal for the relocation of the CA, replacing it with distribution centers (CDs) located through a mathematical model of optimization in strategic locations. As a first approximation for the location of the CDs, thirty neighborhoods were taken from the city, as a sample in the study, and by means of a survey in the population, the

\section{Referencias}

Chopra, S, P Meindl., 2016. Supply Chain Management: Strategic, Planning and Operation, Sixth Edition, Pearson.

Clemen, R. T. and Reilly, T. (2001). Making Hard Decision with Decision Tools Suite, Belmont, CA: Duxbury Press

De Neufville, R. and Keeney, R. L. (1972). Use of decision analysis in airport development for Mexico City. In: Analysis of Public Systems, Drake, A. W., Keeney, R. L., and Morse, P. M., eds. Cambridge, MA: MIT Press.

Dekle, J., M. Lavieri, E. Martin, H. Emir-Farinas, and R. Francis. (2005): A Florida County Locates Disaster Recovery Centers. Interfaces 35, no. 2 113-139

Google Earth [WWW Document]. URL https:// earth.google.com/web/,(acceso 10/08/2018)

INEGI [WWW Document]. URL https:// http://cuentame.inegi.org.mx/monografias/informaci on/hgo/poblacion/,(acceso 10/08/2018) maximum distance traveled by consumers willing to travel for the supply of inputs was determined. The model's solution found that there should be four, the least number of CDs located in the neighborhoods of: Santiago Tlapacoya, San Pedro Nopancalco, Lomas de Vista Hermosa and Villas de Pachuca.

Keywords: Optimal localization, Set covering, Distribution centers, Integer programming, Pachuca City.

Kirkwood, C. (1982). A case history of nuclear power plant site selection. Journal of the Operational Research Society, 33, 353-363.

Merkhofer, M. and Keeney, R. (1987). A multi-attribute utility analysis of alternative sites for the disposal of nuclear waste. Risk Analysis, 7, 173-194.

Ravindran, R, D.P. Warsing, 2014. Supply Chain Engineering: Models and Applications. CRC Press.

Taha H. (2017). Investigación de Operaciones. Décima edición, Pearson.

UnoTV (julio, 2017). Central de abastos de Pachuca, en crisis, por falta de servicios. Recuperado el 20-012019 de: https://www.unotv.com/noticias/estados/ hidalgo/detalle/central-abastos-pachuca-crisis-servi cios-188708/.

Winston. W, C. Albrigth. 2018. Practical Management Science. Sixth edition, Cengage. 